\title{
Challenges on the frontier of intracoronary imaging: atherosclerotic plaque macrophage measurement by optical coherence tomography
}

\author{
Atsushi Tanaka \\ Harvard Medical School \\ Massachusetts General Hospital \\ Wellman Center for Photomedicine \\ Boston, Massachusetts 02114
}

\section{Guillermo J. Tearney}

Brett E. Bouma

Harvard Medical School

Massachusetts General Hospital

Harvard-MIT Division of Health Sciences and Technology Wellman Center for Photomedicine

Department of Dermatology

BAR 703

40 Blossom Street

Boston, Massachusetts 02138

\begin{abstract}
Cellularity of the fibrous caps of coronary atheromas, manifested by the infiltration of macrophages (average size, 20 to $30 \mu \mathrm{m}$ ), is thought to weaken the structural integrity of the cap and predispose plaques to rupture. Therefore, an imaging technology capable of identifying macrophages within fibroatheroma caps in patients could provide valuable information for assessing plaque rupture risk. Recently, intravascular optical coherence tomography (OCT), a high-resolution coronary imaging modality, with an axial resolution of $\sim 10 \mu \mathrm{m}$, has been introduced into the clinical setting. OCT images of the microstructure of the coronary artery wall enable accurate plaque-type characterization, supported by histopathological comparison data. Because of its high resolution, OCT may also be used to identify macrophages in vivo. In this paper we review recent developments in OCT for measuring macrophages in atherosclerotic plaques. () 2010 Society of Photo-Optical Instrumentation Engineers. [DOI: 10.1117/1.3290810]
\end{abstract}

Keywords: optical imaging; optical coherence tomography; macrophage; coronary imaging; vulnerable plaque; acute coronary syndrome.

Paper 09468SSR received Oct. 15, 2009; revised manuscript received Nov. 9, 2009; accepted for publication Nov. 11, 2009; published online Mar. 2, 2010.

\section{Introduction}

Until recently, invasive coronary angiography has been the gold-standard imaging modality for evaluating coronary atherosclerosis. ${ }^{1}$ Angiography, however, provides information only about the lumen but not of the artery wall itself. When grayscale intravascular ultrasound (IVUS) was introduced in early the $1990 \mathrm{~s},{ }^{2,3}$ many cardiologists, especially interventional cardiologists, were fascinated by this device because it allowed the coronary plaque to be directly observed in human patients. Because of its capability to interrogate arterial wall structure, IVUS has been applied for the optimization of coronary angioplasty and has been useful for improving our understanding of the pathophysiology of coronary atherosclerosis. ${ }^{4,5}$ Even so, its resolution $(\sim 100 \mu \mathrm{m})$ and limited contrast prohibits the investigation of many important plaque components, i.e., thin fibrous cap or lipid pool. To address this need, alternative diagnostic modalities have been developed, including spectral analysis of IVUS radio-frequency data (virtual histology IVUS), ${ }^{6}$ integrated backscatter IVUS, ${ }^{7,8}$ coronary angioscopy, ${ }^{9,10}$ elastography, ${ }^{11}$ near-infrared (NIR) spectroscopy, ${ }^{12}$ fluorescence spectroscopy, ${ }^{13}$ and Raman spectroscopy. ${ }^{14}$ For tissue characterization, however, these methods have not supplanted grayscale IVUS due to their low spatial resolutions.

Address all correspondence to Guillermo I. Tearney, PhD, Harvard Medical School, Massachusetts General Hospital, Harvard-MIT Division of Health Science and Technology, Wellman Center for Photomedicine, Department of Dermatology, BAR 703, 40 Blossom Street, Boston, MA 02138. Fax: 617-726-4103; E-mail: gtearney@partners.org
Recently, optical coherence tomography (OCT) has been introduced into the clinical setting as a high-resolution intracoronary imaging modality. OCT can provide information about the microstructure of the coronary artery and has been shown to be capable of detailed tissue characterization, supported by histopathological comparison data. ${ }^{15}$ As such, OCT holds promise for providing a greater understanding of the pathophysiology of coronary artery disease in patients.

\section{Plaque Rupture and ACS}

Drug-eluting stents (DESs) dramatically reduce the rate of restenosis or target-vessel revascularization after percutaneous coronary intervention (PCI) as compared to bare-metal stents (BMS). ${ }^{16,17}$ According to the COURAGE trial conducted in the DES era, however, there were no significant differences between the PCI group and the medical therapy group in terms of major adverse coronary events (MACE), including endpoints of death, myocardial infarction (MI), and hospitalization for acute coronary syndrome (ACS) or MI. ${ }^{18}$ These data suggest that local treatment by PCI as an initial management strategy in patients with stable coronary artery disease does not reduce MACE, beyond that of optimal medical therapy. ${ }^{18}$ Thus, to improve an individual patient's prognosis, we should address the prevention of ACS, including myocardial infarction, unstable angina, and sudden cardiac death, that is currently thought to be mainly caused by plaque rupture. ${ }^{19}$

1083-3668/2010/15(1)/011104/8/\$25.00 @ 2010 SPIE 


\section{Vulnerable Plaque}

Due to the lack of a suitable animal model for atherosclerotic plaque rupture, postmortem study and vascular biology have been the main avenues for advancing our understanding of the pathogenesis of ACS. Postmortem studies revealed that myocardial infarction results from an acute process of plaque rupture or erosion of modestly stenotic plaque. ${ }^{20}$ These findings suggest that invasive coronary angiography alone cannot be used to predict plaque rupture, as the degree of lesion stenosis is not necessarily predictive of plaque rupture risk. The pathologic characteristics of the majority of vulnerable plaques include a thin fibrous cap $(>65 \mu \mathrm{m})$, large lipid pool, and increased macrophage activity. ${ }^{21-24}$ These rupture-related macrophages are active, indicating ongoing inflammation at the site of plaque disruption. ${ }^{19,22,24}$ Macrophages are capable of degrading the extracellular matrix by phagocytosis, by secreting proteolytic enzymes such as plasminogen activators and the family of matrix metalloproteinases, or by reducing collagen synthesis and enhancing smooth muscle cell apoptosis that may weaken the fibrous cap, predisposing it to rupture. $^{12,24-26}$ These cellular and molecular changes appear most prominent at the plaque shoulder, where mechanical strain is also high. ${ }^{27,28}$ This data indicates that macrophages are key cells that precipitate the progression and instability of atherosclerotic coronary plaques.

\section{What Is OCT?}

The most common form of intracoronary OCT has been timedomain OCT (TD-OCT) ${ }^{29-38}$ With TD-OCT, broadband light is split into a reference path, which usually terminates at a reference mirror, and a sample path that includes the coronary catheter. Light returned from both paths is recombined, detected, and digitized. When the reference and sample path lengths are matched to within the coherence length of light, typically approximately $10 \mu \mathrm{m}$ in air, interference with an amplitude that is proportional to the sample's reflectivity at a specific spatial position is detected. By moving the reference path length, reflectivity as a function of depth from within the tissue is obtained by demodulating the fringe pattern as a function of time. This depth-resolved reflectivity profile is commonly termed an A-line. An intracoronary OCT image is obtained by scanning the beam across the sample using a rotational catheter, while storing A-lines at each scan location. For intracoronary OCT, broadband light in the near-infrared range with a wavelength centered around $1300 \mathrm{~nm}$ is utilized, providing an axial resolution of $\sim 10 \mu \mathrm{m}$, a transverse resolution of $20-40 \mu \mathrm{m}$, and a penetration depth that ranges between 1 to $2 \mathrm{~mm}$.

While TD-OCT has been successfully used in many patients for research purposes, ${ }^{15,39-43}$ it is somewhat cumbersome to operate in the catheterization laboratory because blood needs to be purged from the field of view in order to clearly visualize the coronary wall. Intermittent nonocclusive saline purging or proximal balloon occlusion can provide unobstructed views of the artery; $;^{40,42}$ however, these approaches are not desirable for everyday clinical use because of safety and diagnostic yield concerns.

The recent advent of second-generation OCT, also known as frequency-domain OCT (FD-OCT), optical frequencydomain imaging (OFDI), ${ }^{44}$ or swept-source OCT (SS-OCT), ${ }^{45}$ has solved this problem, making it far more practical to conduct intracoronary OCT in the clinical setting. ${ }^{46}$ With FDOCT, the light source is a narrowband laser that rapidly tunes its wavelength over a broad spectral width. As opposed to TD-OCT, the reference path length remains fixed; the interference pattern is therefore detected as function of wavelength. Once the laser has completed a full spectral sweep, a Fourier transform is applied to the spectral interference pattern to obtain an A-line. FD-OCT is advantageous over TDOCT because it has a higher sensitivity and therefore is capable of providing images at a much greater rate $(100$ frames $/ \mathrm{s}){ }^{47}$ This increased speed allows an entire artery to be imaged by OCT during a brief, nonocclusive saline/ radiocontrast purge and rapid helical pullback of the catheter's internal optics.

\section{In Vivo Imaging for Plaque Rupture}

Clinical IVUS studies in patients with ACS have identified asymptomatic disrupted plaques, remote from locations of primary stenosis. ${ }^{48-51}$ These findings support the hypothesis that inflammation is associated with human plaque instability. Additionally, high-sensitivity C-reactive protein (hs-CRP) has been reported as a useful marker for predicting future cardiovascular events. ${ }^{52,53}$ However, the significance of elevated hsCRP levels for coronary atherosclerosis had been unclear. An IVUS study demonstrated the relationship between the elevated hs-CRP levels and the presence of plaque rupture in patients with $\mathrm{ACS}^{54}$ Moreover, serum matrix metalloproteinase- 9 levels, one enzyme that has been implicated in the degradation of the cap's extracellular matrix, are elevated in ACS patients with IVUS evidence of plaque rupture, but not in ACS patients without plaque rupture or patients with stable angina. ${ }^{55}$ Even though these correlations exist, remotesite ruptured plaques detected by IVUS have not yet been found to be related to future events. ${ }^{56}$

\section{Criteria for Vulnerable Plaque}

Detection and treatment of vulnerable plaque that is prone to cause rupture or cardiovascular events has been proposed and is expected to improve management of patients with ACS. ${ }^{57}$ The following major criteria were proposed for detection of a vulnerable plaque. ${ }^{57}$ The presence of one or a combination of these factors may warrant higher risk of plaque complication.

1. Active inflammation. Plaques with active inflammation may be identified by extensive macrophage accumulation. $^{58}$

2. A thin cap with a large lipid core. These plaques have a cap thickness of $<100 \mu \mathrm{m}$ and a lipid core accounting for $>40 \%$ of the plaque's total volume. ${ }^{22,59}$

3. Endothelial denudation with superficial platelet aggregation. These plaques are characterized by superficial erosion and platelet aggregation or fibrin deposition. .2,23,60 $^{2}$

4. Fissured/injured plaque. Plaques with a fissured cap (most of them involving a recent rupture) that did not result in occlusive thrombi may be prone to subsequent thrombosis, entailing occlusive thrombi or thromboemboli. ${ }^{22}$

5. Severe stenosis. On the surface of plaques with severe 


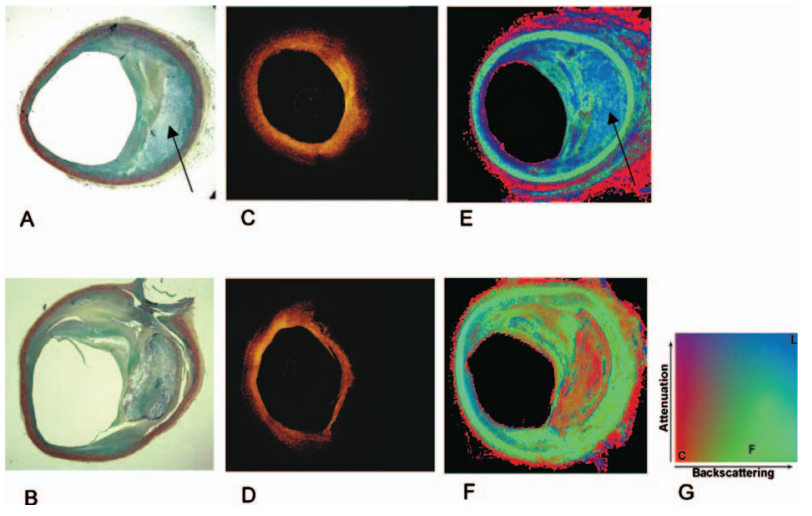

Fig. 1 Tissue characterization by measuring backscattering and attenuation coefficients in OCT. (a) and (b) Histology: Movat's pentachrome staining for a representative fibrolipid plaque (a) and a fibrocalcific plaque (b). An arrow shows necrotic core that contained a large amount of form cells/macrophages. (c) and (d) Corresponding rotary OCT image of (a) and (b). It is difficult to distinguish the three plaque types based on OCT images alone. (e) and (f) Images combining backscattering and attenuation measurement using the color map defined in (g). Necrotic core (arrow) shows the increase in both the backscattering and attenuation coefficients. (g) Color map for combining the backscattering and attenuation measurements. Labels C, F, and $\mathrm{L}$ denote the positions of the average backscattering and attenuation coefficients for calcific, fibrous, and lipid tissue, respectively (Ref. 65).

stenosis, shear stress imposes a significant risk of thrombosis and sudden occlusion. ${ }^{61}$ Therefore, a stenotic plaque may be a vulnerable plaque regardless of ischemia. Moreover, astenotic plaque may indicate the presence of many nonstenotic or less stenotic plaques that can be vulnerable to rupture and thrombosis. $^{62}$

All of the preceding criteria except active inflammation are architectural morphological features of ruptured plaque. Given its $10-\mu \mathrm{m}$ resolution, OCT has the potential capability to visualize many of these features, a notion supported in previous histopathologic correlative studies. ${ }^{41-43,63}$

\section{Ex Vivo Study of Macrophage Imaging by OCT}

Although OCT can identify major morphological features of vulnerable plaque, it has been unclear as to whether OCT has sufficient spatial resolution to enable the identification of macrophages in plaques. Macrophages/foam cells in atherosclerotic plaques are relatively large $(20$ to $30 \mu \mathrm{m})$ and may contain multiple intracellular, lipid-rich phagolysozomes. Because of their size and the refractive index difference between the phagolysozomes and the surrounding intraceulluar fluid, investigators have postulated that macrophages may have a higher OCT signal than surrounding tissue. ${ }^{64}$ Schmitt et al. stated in their paper that macrophage/form cells, which can be either dispersed or clustered, change the optical properties of tissue dramatically. In general, the form cells were found to increase both the backscattering and attenuation coefficients (Fig. 1). ${ }^{65}$

Early comparisons between OCT and histology were carried out to investigate the possibility that OCT may indeed

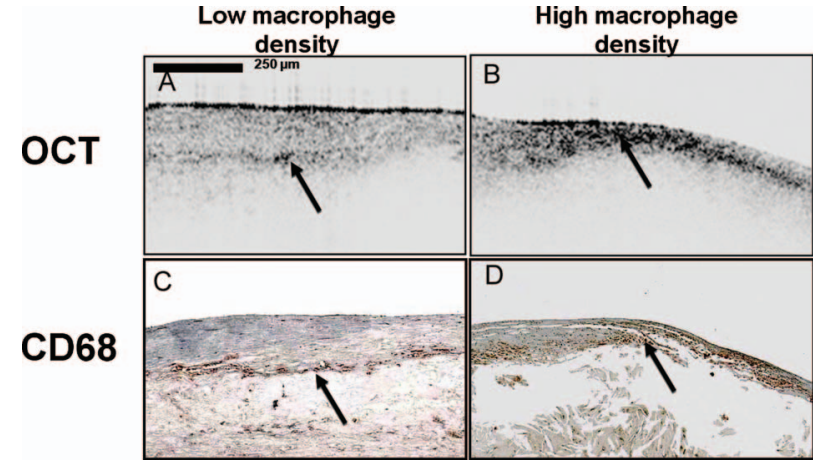

Fig. 2 (a) Raw OCT images of a fibroatheroma with a relatively low number of macrophages within the fibrous cap. (b) Raw OCT images of a fibroatheroma with a high density of macrophages within the fibrous cap. (c) Corresponding histology for (a) (CD68 immunoperoxidase; original magnification $\times 100)$. (d) Corresponding histology for (b) (CD68 immunoperoxidase; original magnification $\times 100$ ) Macrophages [arrows in figure (c) and (d)] could be observed as punctate, highly reflecting regions [arrows in (a) and (b)] in raw OCT images (Ref. 64).

identify these cells in individual lesions and potentially even enable the quantification of macrophage content within the cross-sectional image. ${ }^{64}$ Below, we describe this study in detail. Because the dynamic range of an OCT image is too high (100 to $110 \mathrm{~dB}$ or 10 to 11 orders of magnitude) to be displayed on a standard monitor, the signal range of most OCT images is compressed by taking the base 10 logarithm of the OCT image before display. To evaluate macrophage imaging by OCT, the capabilities of both the raw (linear) OCT data and the logarithm of the OCT data for quantifying macrophage content within fibrous caps were investigated. ${ }^{64}$ To calculate macrophage content, the OCT data within a defined region of interest (ROI) were preprocessed using median filtering to remove background noise and reduce speckle. Within the ROI, the OCT signal was characterized by use of the normalized standard deviation (NSD): ${ }^{64}$

$$
\mathrm{NSD}=\frac{\sigma}{\left(S_{\max }-S_{\min }\right)},
$$

where $\sigma$ is the standard deviation of the OCT signal, $S_{\max }$ is the maximum OCT image value, and $S_{\min }$ is the minimum OCT image value (Fig. 2).

OCT NSD measurements were compared with histologic quantification of macrophage density, estimated by use of immunohistochemical staining with CD68 (Ref. 64). A total of 26 lipid-rich plaques were processed in this manner. The cap macrophage density was quantified morphometrically by calculating the percent area of CD68 staining. Smooth muscle cell densities were also compared to NSD using morphometric analysis of smooth muscle actin stained slides. The area percentage of CD68 and smooth muscle actin staining was compared to the NSD of the OCT signal intensity within corresponding regions of interest. The results of this analysis showed that a homogenous OCT signal corresponded to a low macrophage density and a heterogeneous OCT signal with punctate, highly reflecting regions corresponded to high macrophage content (Fig. 3). 


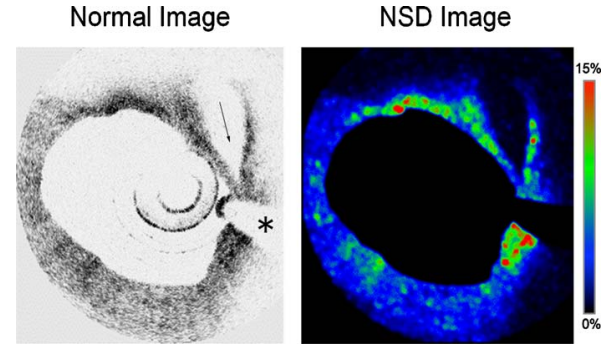

Fig. 3 Conventional OCT image and normarlized standard deviation (NSD) image. An NSD image was computed for each pixel within the cap and displayed by use of a color lookup table. The arrow denotes a site of disruption within a lipid-rich plaque. The asterisk $(*)$ is an artifact by the guide wire.

The macrophage density, as determined by immunohistochemistry, correlated with the base 10 logarithm OCT NSD $(r=0.47, P<0.05)$ and with the linear OCT NSD $(r=0.84$, $P<0.0001$ ) (Ref. 64; Fig. 4).

The partial correlation of linear OCT NSD and CD68 percent staining, controlling for cap thickness, was $r=0.80$ $(P<.0001)$, indicating that the OCT measurement of macrophage density was independent of cap thickness. OCT NSD was inversely correlated to smooth muscle actin staining, reflecting the inverse relationship between macrophages and smooth muscle cells. These results highlighted the ability of OCT to detect and quantify macrophage infiltration. The simplicity of the algorithm used for macrophage evaluation makes it possible for this information to be superimposed on the standard OCT image in real time. ${ }^{64}$

\section{In Vivo Macrophage Imaging}

The demonstration that OCT can potentially image macrophages ex vivo raised interest as to whether this technique could be also applied in human patients. To evaluate the clinical significance of OCT macrophage density (NSD) quantification, intracoronary OCT was conducted at culprit and nonculprit coronary plaques in a series of 49 patients undergoing percutaneous coronary intervention. ${ }^{41}$ The patients were di-

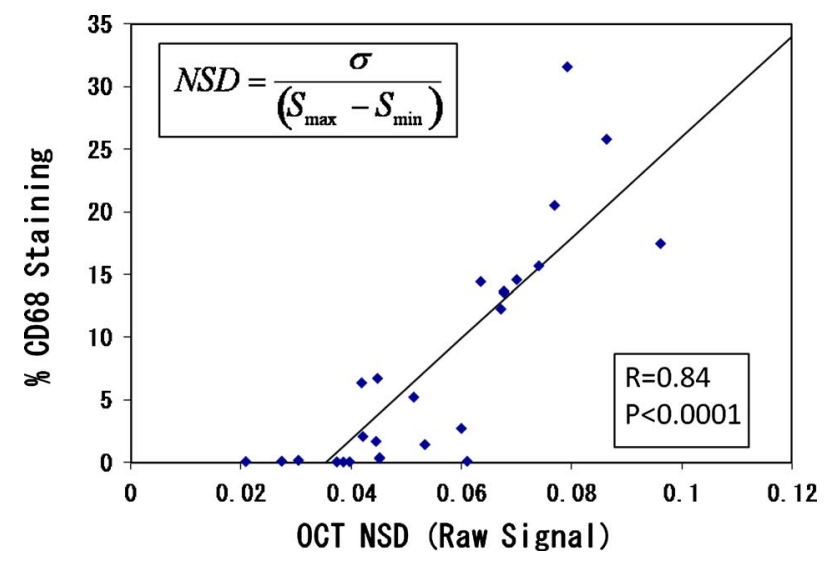

Fig. 4 Correlation between raw OCT NSD data and \% area staining of CD68. The macrophage density, as determined by immunohistochemistry, correlated with the raw OCT data $(r=0.84, P>0.0001)$ (Ref. 64).

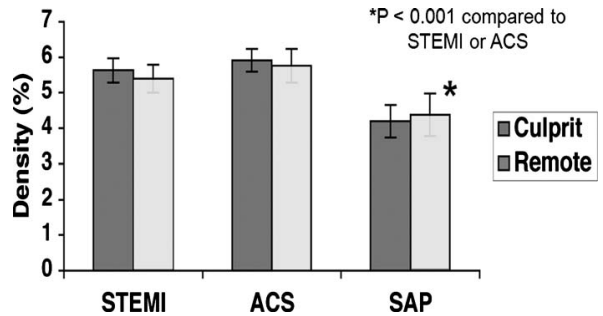

Fig. 5 Clinical presentations versus NSD measurement of macrophage density. NSD was elevated in patients presenting with STEMI or ACS compared with SAP. No difference in the macrophage concentration was found between the STEMI patients and the ACS patients (Ref. 41)

vided into three groups: (1) ST-elevation myocardial infarction (STEMI), $n=19$; (2) acute coronary syndrome (ACS), which incorporates unstable angina and non-STEMI, $n=19$; and (3) stable angina pectoris (SAP), $n=11$.

Results from this study demonstrated a significant relationship between the clinical presentation and the macrophage concentration, with a greater concentration of macrophages in patients presenting with STEMI or ACS compared with SAP. No statistically significant difference in the macrophage concentration was found between the STEMI patients and the ACS patients or between remote and culprit sites for each clinical group. (Fig. 5).

For fibrous plaques, the macrophage density of the unstable group was found to be significantly higher than that of the stable group. This finding suggested that macrophage infiltration was not confined to just lipid-rich plaque but was present throughout the coronary tree. Although fibrous plaques have traditionally been considered to be more stable, erosion of the superficial endothelial layer overlying pathological intimal thickening is found in some patients with an acute coronary event (Fig. 6). ${ }^{23,60}$

A statistically significant higher macrophage density was found at rupture sites compared with the adjacent nonruptured cap within the same image. Moreover, macrophage density at rupture sites was significantly greater than that of all nonrup-

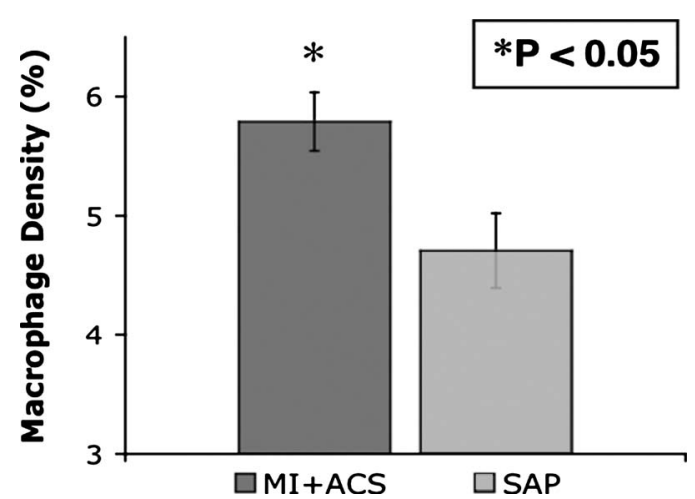

Fig. 6 Macrophage density in fibrous plaques. Even in fibrous plaque, macrophage density of unstable patients was significantly higher than that of the stable patients. This finding suggested that macrophage infiltration was not confined to just lipid-rich plaque but was evident throughout the coronary tree (Ref. 41). 


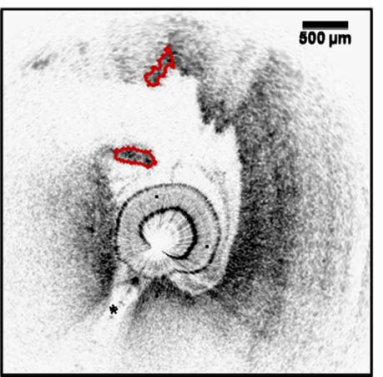

(a)

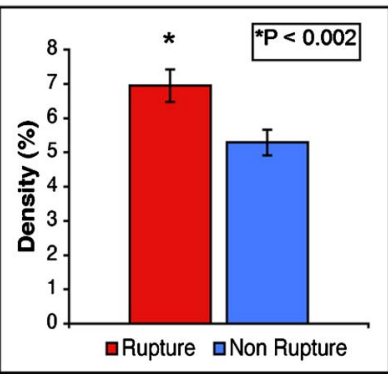

(b)
Fig. 7 Fibrous cap macrophage density at rupture sites. (a) Macrophages are elevated at the rupture site in a representative ruptured plaque seen by OCT. OCT clearly shows that the fibrous cap disrupted at $11 \mathrm{o}^{\prime}$ clock. The $*$ represents the guide wire shadow. (b) The column plot shows that macrophage density at rupture sites (left column), measured by OCT NSD, was significantly higher than adjacent nonruptured sites (right column).

tured culprit sites in the combined STEMI and ACS groups (Fig. 7).

The spatial distribution of macrophages within the fibrous cap of lipid-rich plaques was examined to determine the relationship between the proximity of macrophages to the endothelial surface and coronary syndrome. The fibrous cap was divided into a superficial layer $(<50 \mu \mathrm{m}$ from the luminal surface) and a subsurface layer $(>50 \mu \mathrm{m}$ from the luminal surface). For each layer, a receiver operator characteristic (ROC) curve was computed for the prediction of an unstable coronary presentation. At culprit sites, the area under the ROC curve for superficial macrophage density was significantly greater than the area under the ROC curve of the subsurface macrophage density. There was no such statistically significant difference seen at the nonculprit sites. This result indicated that macrophages at the surface of these culprit lesions were predictive of clinical syndrome (Ref. 41).

\section{Clinical Application of Macrophage Imaging}

To compensate for the decrease in coronary blood flow caused by atheromatous plaque growth, the coronary wall apparently responds by outward expansion of the artery, termed positive (compensatory) remodeling. ${ }^{65}$ Paradoxically, autopsy studies have shown that this response is often found in lesions from patients who presented with ACS. ${ }^{66}$ Recently, a few OCT studies have reported that positive remodeling is more commonly associated with lipid-rich plaque, a thin fibrous cap, the presence of thin-cap fibroatheroma (TCFA) and a larger inflammatory cell infiltrate, or an elevated hs-CRP. ${ }^{67,68}$ These papers have also reported that fibrous cap thickness is inversely correlated with the remodeling index. One group reported that the remodeling index was positively correlated with plaque fibrous cap macrophage density ${ }^{67}$ and that the plaque fibrous cap thickness was inversely correlated with plaque fibrous cap macrophage density (Fig. 8). These human data support the notion that plaque fibrous cap macrophage density may be useful for predicting future ACS events.

\section{Limitations}

It is important to acknowledge the limitations of macrophage imaging by OCT. First and foremost, the NSD parameter has been utilized by only one research group. ${ }^{64,67,69}$ Ongoing efforts to standardize this metric and other interpretation criteria is essential in this field to fully leverage the capabilities of this technology. A second issue is that while the NSD metric is simple to understand, it is also limited. First, the NSD parameter is not the same as macrophage density; OCT images without macrophages inherently have a nonzero NSD due to baseline scattering and residual speckle. Other components, such as the internal elastic lamina, cholesterol crystals, and some calcifications may also give a high NSD value. The NSD parameter was developed and evaluated only in the context of fibroatheroma caps, and therefore caution should be taken when applying this measurement to other areas of the coronary wall. Future research should be conducted to de-

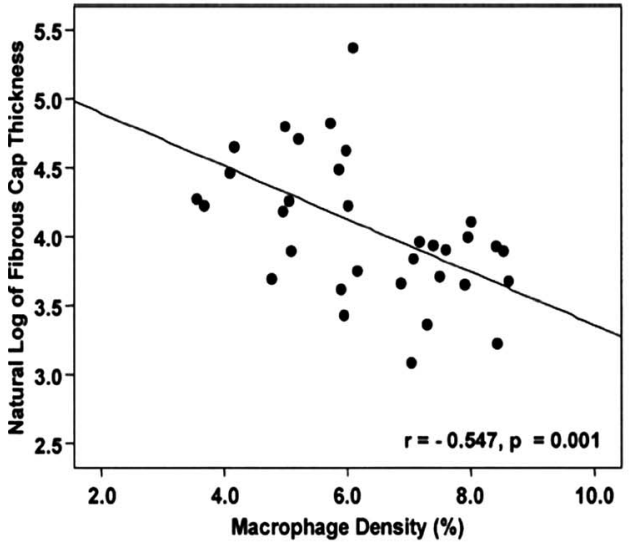

(a)

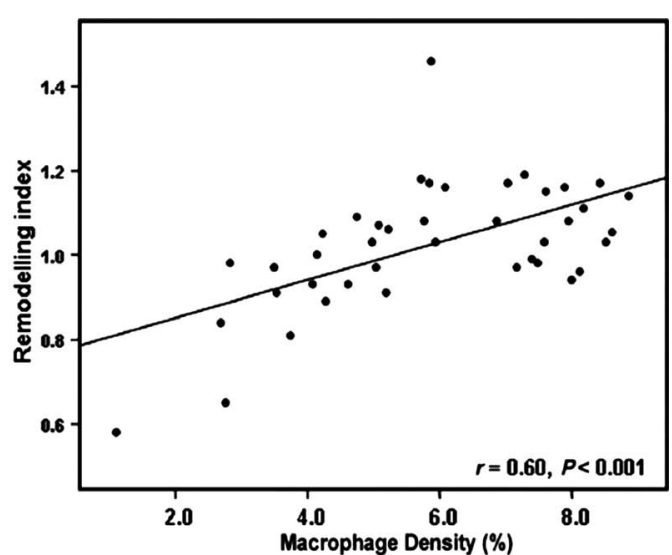

(b)

Fig. 8 Correlation between macrophage density and fibrous cap thickness or remodeling index. Scatter plots of (a) macrophage density to the logarithm of fibrous cap thickness and (b) macrophage density to remodeling. Pearson's correlation coefficient ( $r$ ) and the $P$-value are shown (Ref. 67). 
velop an improved OCT macrophage metric that can be more generally applied to the entire artery.

\section{Conclusions}

The possibility of seeing macrophages in a cross-sectional image is very exciting for interventional cardiology and could open opportunities for studying these cells in patients and diagnosing vulnerable plaques. Research conducted in this area, including the development of the NSD parameter, is early but promising. OCT measurements of NSD appear to correlate with histopathologic markers of macrophages. Furthermore, macrophage measurements obtained from patients support hypotheses about macrophage content derived from autopsy and vascular biology studies. These results provide an impetus to improve this parameter further, standardize OCT measurements of macrophages, and conduct additional validation studies, so that macrophage information can be widely utilized in interventional cardiology research and clinical practice.

\section{References}

1. W. L. Proudfit, E. K. Shirey, and F. M. Sones Jr., "Selective cine coronary arteriography. Correlation with clinical findings in 1,000 patients," Circulation 33(6), 901-910 (1966).

2. P. G. Yock, D. T. Linker, and B. A. Angelsen, "Two-dimensional intravascular ultrasound: technical development and initial clinical experience," J. Am. Soc. Echocardiogr 2(4), 296-304 (1989).

3. B. N. Potkin, A. L. Bartorelli, J. M. Gessert, R. F. Neville, Y. Almagor, W. C. Roberts, and M. B. Leon, "Coronary artery imaging with intravascular high-frequency ultrasound," Circulation 81(5), 15751585 (1990).

4. K. Sudhir, P. J. Fitzgerald, J. S. MacGregor, T. DeMarco, T. A. Ports, K. Chatterjee, and P. G. Yock, "Transvenous coronary ultrasound imaging. A novel approach to visualization of the coronary arteries," Circulation 84(5), 1957-1961 (1991).

5. S. E. Nissen and P. Yock, "Intravascular ultrasound: novel pathophysiological insights and current clinical applications," Circulation 103(4), 604-616 (2001).

6. A. Nair, B. D. Kuban, E. M. Tuzcu, P. Schoenhagen, S. E. Nissen, and D. G. Vince, "Coronary plaque classification with intravascular ultrasound radiofrequency data analysis," Circulation 106(17), 22002206 (2002).

7. J. C. Machado and F. S. Foster, "Ultrasonic integrated backscatter coefficient profiling of human coronary arteries in vitro," IEEE Trans. Ultrason. Ferroelectr. Freq. Control 48(1), 17-27 (2001).

8. M. Kawasaki, H. Takatsu, T. Noda, K. Sano, Y. Ito, K. Hayakawa, K. Tsuchiya, M. Arai, K. Nishigaki, G. Takemura, S. Minatoguchi, T. Fujiwara, and H. Fujiwara, "In vivo quantitative tissue characterization of human coronary arterial plaques by use of integrated backscatter intravascular ultrasound and comparison with angioscopic findings," Circulation 105(21), 2487-2492 (2002).

9. K. Mizuno, A. Miyamoto, K. Satomura, A. Kurita, T. Arai, M. Sakurada, S. Yanagida, and H. Nakamura, "Angioscopic coronary macromorphology in patients with acute coronary disorders," Lancet 337(8745), 809-812 (1991).

10. T. Thieme, K. D. Wernecke, R. Meyer, E. Brandenstein, D. Habedank, A. Hinz, S. B. Felix, G. Baumann, and F. X. Kleber, "Angioscopic evaluation of atherosclerotic plaques: validation by histomorphologic analysis and association with stable and unstable coronary syndromes," J. Am. Coll. Cardiol. 28(1), 1-6 (1996).

11. M. Naghavi, M. Madjid, M. R. Khan, R. M. Mohammadi, J. T. Willerson, and S. W. Casscells, "New developments in the detection of vulnerable plaque," Curr. Atheroscler. Rep. 3(2), 125-135 (2001).

12. P. R. Moreno, R. A. Lodder, K. R. Purushothaman, W. E. Charash, W. N. O'Connor, and J. E. Muller, "Detection of lipid pool, thin fibrous cap, and inflammatory cells in human aortic atherosclerotic plaques by near-infrared spectroscopy," Circulation 105(8), 923-927 (2002).

13. L. Marcu, M. C. Fishbein, J. M. Maarek, and W. S. Grundfest, "Discrimination of human coronary artery atherosclerotic lipid-rich le- sions by time-resolved laser-induced fluorescence spectroscopy," Arterioscler., Thromb., Vasc. Biol. 21(7), 1244-1250 (2001).

14. T. J. Romer, J. F. Brennan 3rd, M. Fitzmaurice, M. L. Feldstein, G. Deinum, J. L. Myles, J. R. Kramer, R. S. Lees, and M. S. Feld, "Histopathology of human coronary atherosclerosis by quantifying its chemical composition with Raman spectroscopy," Circulation 97(9), 878-885 (1998).

15. G. J. Tearney, I. K. Jang, and B. E. Bouma, "Optical coherence tomography for imaging the vulnerable plaque," J. Biomed. Opt. 11(2), 021002 (2006).

16. M. C. Morice, P. W. Serruys, J. E. Sousa, J. Fajadet, E. Ban Hayashi, M. Perin, A. Colombo, G. Schuler, P. Barragan, G. Guagliumi, F. Molnar, and R. Falotico, "A randomized comparison of a sirolimuseluting stent with a standard stent for coronary revascularization," $N$. Engl. J. Med. 346(23), 1773-1780 (2002).

17. J. W. Moses, M. B. Leon, J. J. Popma, P. J. Fitzgerald, D. R. Holmes, C. O"Shaughnessy, R. P. Caputo, D. J. Kereiakes, D. O. Williams, P. S. Teirstein, J. L. Jaeger, and R. E. Kuntz, "Sirolimus-eluting stents versus standard stents in patients with stenosis in a native coronary artery," N. Engl. J. Med. 349(14), 1315-1323 (2003).

18. W. E. Boden, R. A. O'Rourke, K. K. Teo, P. M. Hartigan, D. J. Maron, W. J. Kostuk, M. Knudtson, M. Dada, P. Casperson, C. L. Harris, B. R. Chaitman, L. Shaw, G. Gosselin, S. Nawaz, L. M. Title, G. Gau, A. S. Blaustein, D. C. Booth, E. R. Bates, J. A. Spertus, D. S. Berman, G. B. Mancini, and W. S. Weintraub, "Optimal medical therapy with or without PCI for stable coronary disease," N. Engl. J. Med. 356(15), 1503-1516 (2007).

19. V. Fuster, L. Badimon, M. Cohen, J. A. Ambrose, J. J. Badimon, and J. Chesebro, "Insights into the pathogenesis of acute ischemic syndromes," Circulation 77(6), 1213-1220 (1988).

20. E. Falk, "Plaque rupture with severe pre-existing stenosis precipitating coronary thrombosis. Characteristics of coronary atherosclerotic plaques underlying fatal occlusive thrombi," Br. Heart J. 50(2), 127134 (1983).

21. M. J. Davies, P. D. Richardson, N. Woolf, D. R. Katz, and J. Mann, "Risk of thrombosis in human atherosclerotic plaques: role of extracellular lipid, macrophage, and smooth muscle cell content," $\mathrm{Br}$ Heart J. 69(5), 377-381 (1993).

22. R. Virmani, F. D. Kolodgie, A. P. Burke, A. Farb, and S. M. Schwartz, "Lessons from sudden coronary death: a comprehensive morphological classification scheme for atherosclerotic lesions," Arterioscler., Thromb., Vasc. Biol. 20(5), 1262-1275 (2000).

23. A. C. van der Wal, A. E. Becker, C. M. van der Loos, and P. K. Das, "Site of intimal rupture or erosion of thrombosed coronary atherosclerotic plaques is characterized by an inflammatory process irrespective of the dominant plaque morphology," Circulation 89(1), 36-44 (1994).

24. P. Libby, "Current concepts of the pathogenesis of the acute coronary syndromes," Circulation 104(3), 365-372 (2001).

25. E. Falk, P. K. Shah, and V. Fuster, "Coronary plaque disruption," Circulation 92(3), 657-671 (1995).

26. E. Arbustini, M. Grasso, M. Diegoli, A. Pucci, M. Bramerio, D Ardissino, L. Angoli, S. de Servi, E. Bramucci, A. Mussini, G. Minzioni, M. Vigano, and G. Specchia, "Coronary atherosclerotic plaques with and without thrombus in ischemic heart syndromes: a morphologic, immunohistochemical, and biochemical study," Am. J. Cardiol. 68(7), 36B-50B (1991).

27. G. C. Cheng, H. M. Loree, R. D. Kamm, M. C. Fishbein, and R. T. Lee, "Distribution of circumferential stress in ruptured and stable atherosclerotic lesions. A structural analysis with histopathological correlation," Circulation 87(4), 1179-1187 (1993).

28. R. T. Lee, H. M. Loree, G. C. Cheng, E. H. Lieberman, N. Jaramillo, and F. J. Schoen, "Computational structural analysis based on intravascular ultrasound imaging before in vitro angioplasty: prediction of plaque fracture locations," J. Am. Coll. Cardiol. 21(3), 777-782 (1993).

29. D. Huang, E. A. Swanson, C. P. Lin, J. S. Schuman, W. G. Stinson, W. Chang, M. R. Hee, T. Flotte, K. Gregory, C. A. Puliafito, and J. G. Fujimoto, "Optical coherence tomography," Science 254(5035), 1178-1181 (1991).

30. I. K. Jang, G. J. Tearney, B. MacNeill, M. Takano, F. Moselewski, N. Iftima, M. Shishkov, S. Houser, H. T. Aretz, E. F. Halpern, and B. E. Bouma, "In vivo characterization of coronary atherosclerotic plaque by use of optical coherence tomography," Circulation 111(12), 15511555 (2005). 
31. D. Matsumoto, J. Shite, T. Shinke, H. Otake, Y. Tanino, D. Ogasawara, T. Sawada, O. L. Paredes, K. Hirata, and M. Yokoyama, "Neointimal coverage of sirolimus-eluting stents at 6-month followup: evaluated by optical coherence tomography," Eur. Heart J. 28(8), 961-967 (2007).

32. M. Takano, S. Inami, I. K. Jang, M. Yamamoto, D. Murakami, K. Seimiya, T. Ohba, and K. Mizuno, "Evaluation by optical coherence tomography of neointimal coverage of sirolimus-eluting stent three months after implantation," Am. J. Cardiol. 99(8), 1033-1038 (2007).

33. J. Tanigawa, P. Barlis, and C. Di Mario, "Intravascular optical coherence tomography: optimisation of image acquisition and quantitative assessment of stent strut apposition," EuroIntervention 3(1), 128-136 (2007).

34. S. Tanimoto, G. Rodriguez-Granillo, P. Barlis, S. de Winter, N. Bruining, R. Hamers, M. Knappen, S. Verheye, P. W. Serruys, and E. Regar, "A novel approach for quantitative analysis of intracoronary optical coherence tomography: high inter-observer agreement with computer-assisted contour detection," Catheter Cardiovasc. Interv. 72(2), 228-235 (2008)

35. F. Prati, M. Cera, V. Ramazzotti, F. Imola, R. Giudice, M. Giudice, S. D. Propris, and M. Albertucci, "From bench to bedside: a novel technique of acquiring OCT images," Circ. J. 72(5), 839-843 (2008).

36. A. Tanaka, T. Imanishi, H. Kitabata, T. Kubo, S. Takarada, T. Tanimoto, A. Kuroi, H. Tsujioka, H. Ikejima, K. Komukai, H. Kataiwa, K. Okouchi, M. Kashiwaghi, K. Ishibashi, H. Matsumoto, K. Takemoto, N. Nakamura, K. Hirata, M. Mizukoshi, and T. Akasaka, "Lipid-rich plaque and myocardial perfusion after successful stenting in patients with non-ST-segment elevation acute coronary syndrome: an optical coherence tomography study," Eur. Heart J. 30(11), 13481355 (2009).

37. N. Gonzalo, P. W. Serruys, T. Okamura, H. M. van Beusekom, H. M. Garcia-Garcia, G. van Soest, W. van der Giessen, and E. Regar, "Optical coherence tomography patterns of stent restenosis," Am. Heart $J$. 158(2), 284-293 (2009).

38. G. Guagliumi and M. Costa, "From a foggy sight to a clear vision," JACC Cardiovasc. Interv. 2(5), 467-469 (2009).

39. B. E. Bouma, G. J. Tearney, H. Yabushita, M. Shishkov, C. R. Kauffman, D. DeJoseph Gauthier, B. D. MacNeill, S. L. Houser, H. T Aretz, E. F. Halpern, and I. K. Jang, "Evaluation of intracoronary stenting by intravascular optical coherence tomography," Heart 89(3), 317-320 (2003)

40. H. Yabushita, B. E. Bouma, S. L. Houser, H. T. Aretz, I. K. Jang, K. H. Schlendorf, C. R. Kauffman, M. Shishkov, D. H. Kang, E. F. Halpern, and G. J. Tearney, "Characterization of human atherosclerosis by optical coherence tomography," Circulation 106(13), 1640 1645 (2002)

41. B. D. MacNeill, I. K. Jang, B. E. Bouma, N. Iftimia, M. Takano, H. Yabushita, M. Shishkov, C. R. Kauffman, S. L. Houser, H. T. Aretz, D. DeJoseph, E. F. Halpern, and G. J. Tearney, "Focal and multi-focal plaque macrophage distributions in patients with acute and stable presentations of coronary artery disease," J. Am. Coll. Cardiol. 44(5), 972-979 (2004).

42. T. Kubo, T. Imanishi, S. Takarada, A. Kuroi, S. Ueno, T. Yamano, T. Tanimoto, Y. Matsuo, T. Masho, H. Kitabata, K. Tsuda, Y. Tomobuchi, and T. Akasaka, "Assessment of culprit lesion morphology in acute myocardial infarction: ability of optical coherence tomography compared with intravascular ultrasound and coronary angioscopy," $J$. Am. Coll. Cardiol. 50(10), 933-939 (2007).

43. A. Tanaka, T. Imanishi, H. Kitabata, T. Kubo, S. Takarada, H. Kataiwa, A. Kuroi, H. Tsujioka, T. Tanimoto, N. Nakamura, M. Mizukoshi, K. Hirata, and T. Akasaka, "Distribution and frequency of thin-capped fibroatheromas and ruptured plaques in the entire culprit coronary artery in patients with acute coronary syndrome as determined by optical coherence tomography," Am. J. Cardiol. 102(8), 975-979 (2008).

44. S. H. Yun, C. Boudoux, G. J. Tearney, and B. E. Bouma, "High-speed wavelength-swept semiconductor laser with a polygon-scanner-based wavelength filter," Opt. Lett. 28(20), 1981-1983 (2003).

45. M. Choma, M. Sarunic, C. Yang, and J. Izatt, "Sensitivity advantage of swept source and Fourier domain optical coherence tomography," Opt. Express 11(18), 2183-2189 (2003).

46. G. J. Tearney, S. Waxman, M. Shishkov, B. J. Vakoc, M. J. Suter, M. I. Freilich, A. E. Desjardins, W. Y. Oh, L. A. Bartlett, M. Rosenberg, and B. E. Bouma, "Three-dimensional coronary artery microscopy by intracoronary optical frequency domain imaging," JACC Cardiovasc. Imag. 1(6), 752-761 (2008).

47. J. F. de Boer, B. Cense, B. H. Park, M. C. Pierce, G. J. Tearney, and B. E. Bouma, "Improved signal-to-noise ratio in spectral-domain compared with time-domain optical coherence tomography," Opt. Lett. 28(21), 2067-2069 (2003).

48. J. Ge, F. Chirillo, J. Schwedtmann, G. Gorge, M. Haude, D. Baumgart, V. Shah, C. von Birgelen, S. Sack, H. Boudoulas, and R. Erbel, "Screening of ruptured plaques in patients with coronary artery disease by intravascular ultrasound," Heart 81(6), 621-627 (1999).

49. A. Tanaka, T. Kawarabayashi, Y. Nishibori, T. Sano, Y. Nishida, D. Fukuda, K. Shimada, and J. Yoshikawa, "No-reflow phenomenon and lesion morphology in patients with acute myocardial infarction," Circulation 105(18), 2148-2152 (2002).

50. G. Rioufol, G. Finet, I. Ginon, X. Andre-Fouet, R. Rossi, E. Vialle, E. Desjoyaux, G. Convert, J. F. Huret, and A. Tabib, "Multiple atherosclerotic plaque rupture in acute coronary syndrome: a three-vessel intravascular ultrasound study," Circulation 106(7), 804-808 (2002).

51. A. Tanaka, K. Shimada, T. Sano, M. Namba, T. Sakamoto, Y. Nishida, T. Kawarabayashi, D. Fukuda, and J. Yoshikawa, "Multiple plaque rupture and C-reactive protein in acute myocardial infarction," J. Am. Coll. Cardiol. 45(10), 1594-1599 (2005).

52. P. M. Ridker, M. Cushman, M. J. Stampfer, R. P. Tracy, and C. H. Hennekens, "Inflammation, aspirin, and the risk of cardiovascular disease in apparently healthy men," N. Engl. J. Med. 336(14), 973979 (1997).

53. P. M. Ridker, C. H. Hennekens, J. E. Buring, and N. Rifai, "Creactive protein and other markers of inflammation in the prediction of cardiovascular disease in women," N. Engl. J. Med. 342(12), 836$843(2000)$

54. T. Sano, A. Tanaka, M. Namba, Y. Nishibori, Y. Nishida, T. Kawarabayashi, D. Fukuda, K. Shimada, and J. Yoshikawa, "C-reactive protein and lesion morphology in patients with acute myocardial infarction," Circulation 108(3), 282-285 (2003).

55. D. Fukuda, K. Shimada, A. Tanaka, T. Kusuyama, H. Yamashita, S. Ehara, Y. Nakamura, T. Kawarabayashi, H. Iida, M. Yoshiyama, and J. Yoshikawa, "Comparison of levels of serum matrix metalloproteinase-9 in patients with acute myocardial infarction versus unstable angina pectoris versus stable angina pectoris," Am. $J$. Cardiol. 97(2), 175-180 (2006).

56. G. Rioufol, M. Gilard, G. Finet, I. Ginon, J. Boschat, and X. AndreFouet, "Evolution of spontaneous atherosclerotic plaque rupture with medical therapy: long-term follow-up with intravascular ultrasound," Circulation 110(18), 2875-2880 (2004).

57. M. Naghavi, P. Libby, E. Falk, S. W. Casscells, S. Litovsky, J. Rumberger, J. J. Badimon, C. Stefanadis, P. Moreno, G. Pasterkamp, Z. Fayad, P. H. Stone, S. Waxman, P. Raggi, M. Madjid, A. Zarrabi, A. Burke, C. Yuan, P. J. Fitzgerald, D. S. Siscovick, C. L. de Korte, M. Aikawa, K. E. Juhani Airaksinen, G. Assmann, C. R. Becker, J. H. Chesebro, A. Farb, Z. S. Galis, C. Jackson, I. K. Jang, W. Koenig, R. A. Lodder, K. March, J. Demirovic, M. Navab, S. G. Priori, M. D. Rekhter, R. Bahr, S. M. Grundy, R. Mehran, A. Colombo, E. Boerwinkle, C. Ballantyne, W. Insull Jr., R. S. Schwartz, R. Vogel, P. W. Serruys, G. K. Hansson, D. P. Faxon, S. Kaul, H. Drexler, P. Greenland, J. E. Muller, R. Virmani, P. M. Ridker, D. P. Zipes, P. K. Shah, and J. T. Willerson, "From vulnerable plaque to vulnerable patient: a call for new definitions and risk assessment strategies: part I," Circulation 108(14), 1664-1672 (2003).

58. P. Constantinides, "Pathogenesis of cerebral artery thrombosis in man," Arch. Pathol. 83(5), 422-428 (1967).

59. F. D. Kolodgie, A. P. Burke, A. Farb, H. K. Gold, J. Yuan, J. Narula, A. V. Finn, and R. Virmani, "The thin-cap fibroatheroma: a type of vulnerable plaque: the major precursor lesion to acute coronary syndromes," Curr. Opin. Cardiol. 16(5), 285-292 (2001).

60. A. Farb, A. P. Burke, A. L. Tang, T. Y. Liang, P. Mannan, J. Smialek, and R. Virmani, "Coronary plaque erosion without rupture into a lipid core. A frequent cause of coronary thrombosis in sudden coronary death," Circulation 93(7), 1354-1363 (1996).

61. A. Mailhac, J. J. Badimon, J. T. Fallon, A. Fernandez-Ortiz, B. Meyer, J. H. Chesebro, V. Fuster, and L. Badimon, "Effect of an eccentric severe stenosis on fibrin(ogen) deposition on severely damaged vessel wall in arterial thrombosis. Relative contribution of fibrin(ogen) and platelets," Circulation 90(2), 988-996 (1994).

62. J. A. Goldstein, D. Demetriou, C. L. Grines, M. Pica, M. Shoukfeh, 
and W. W. O'Neill, "Multiple complex coronary plaques in patients with acute myocardial infarction," N. Engl. J. Med. 343(13), 915-922 (2000).

63. A. Tanaka, T. Imanishi, H. Kitabata, T. Kubo, S. Takarada, T. Tanimoto, A. Kuroi, H. Tsujioka, H. Ikejima, S. Ueno, H. Kataiwa, K. Okouchi, M. Kashiwaghi, H. Matsumoto, K. Takemoto, N. Nakamura, K. Hirata, M. Mizukoshi, and T. Akasaka, "Morphology of exertion-triggered plaque rupture in patients with acute coronary syndrome: an optical coherence tomography study," Circulation 118(23), 2368-2373 (2008).

64. G. J. Tearney, H. Yabushita, S. L. Houser, H. T. Aretz, I. K. Jang, K. H. Schlendorf, C. R. Kauffman, M. Shishkov, E. F. Halpern, and B. E. Bouma, "Quantification of macrophage content in atherosclerotic plaques by optical coherence tomography," Circulation 107(1), 113119 (2003)

65. S. Glagov, E. Weisenberg, C. K. Zarins, R. Stankunavicius, and G. J. Kolettis, "Compensatory enlargement of human atherosclerotic coronary arteries," N. Engl. J. Med. 316(22), 1371-1375 (1987).

66. A. M. Varnava, P. G. Mills, and M. J. Davies, "Relationship between coronary artery remodeling and plaque vulnerability," Circulation 105(8), 939-943 (2002).

67. O. C. Raffel, F. M. Merchant, G. J. Tearney, S. Chia, D. D. Gauthier, E. Pomerantsev, K. Mizuno, B. E. Bouma, and I. K. Jang, "In vivo association between positive coronary artery remodeling and coronary plaque characteristics assessed by intravascular optical coherence tomography," Eur. Heart J. 29(14), 1721-1728 (2008).

68. M. Kashiwagi, A. Tanaka, H. Kitabata, H. Tsujioka, H. Matsumoto, Y. Arita, K. Ookochi, A. Kuroi, H. Kataiwa, T. Tanimoto, H. Ikejima, S. Takarada, T. Kubo, K. Hirata, N. Nakamura, M. Mizukoshi, T. Imanishi, and T. Akasaka, "Relationship between coronary arterial remodeling, fibrous cap thickness and high-sensitivity C-reactive protein levels in patients with acute coronary syndrome," Circ. J. 73(7), 1291-1295 (2009).

69. O. C. Raffel, G. J. Tearney, D. D. Gauthier, E. F. Halpern, B. E. Bouma, and I. K. Jang, "Relationship between a systemic inflammatory marker, plaque inflammation, and plaque characteristics determined by intravascular optical coherence tomography," Arterioscler. Thromb., Vasc. Biol. 27(8), 1820-1827 (2007). 